\title{
Millimeter and submillimeter wave spectra of ${ }^{13} \mathrm{C}$ methylamine ${ }^{\star}$
}

\author{
R. A. Motiyenko ${ }^{1}$, L. Margulès ${ }^{1}$, V. V. Ilyushin², I. A. Smirnov², E. A. Alekseev², D. T. Halfen², and L. M. Ziurys ${ }^{3}$ \\ ${ }^{1}$ Laboratoire de Physique des Lasers, Atomes, et Molécules, UMR CNRS 8523, Université de Lille 1, \\ 59655 Villeneuve d'Ascq Cédex, France \\ e-mail: roman.motienko@univ-lille1.fr \\ 2 Institute of Radio Astronomy of NASU, Chervonopraporna, 4, 61002 Kharkov, Ukraine \\ 3 Departments of Chemistry and Astronomy, Arizona Radio Observatory and Steward Observatory, University of Arizona, \\ Tucson, AZ 85721, USA
}

Received 9 July 2015 / Accepted 7 December 2015

\section{ABSTRACT}

\begin{abstract}
Context. Methylamine $\left(\mathrm{CH}_{3} \mathrm{NH}_{2}\right)$ is a light molecule of astrophysical interest, which has an intensive rotational spectrum that extends in the submillimeter wave range and far beyond, even at temperatures characteristic for the interstellar medium. It is likely for ${ }^{13} \mathrm{C}$ isotopologue of methylamine to be identified in astronomical surveys, but there is no information available for the ${ }^{13} \mathrm{CH}_{3} \mathrm{NH}_{2}$ millimeter and submillimeter wave spectra.

Aims. In this context, to provide reliable predictions of ${ }^{13} \mathrm{CH}_{3} \mathrm{NH}_{2}$ spectrum in millimeter and submillimeter wave ranges, we have studied rotational spectra of the ${ }^{13} \mathrm{C}$ methylamine isotopologue in the frequency range from 48 to $945 \mathrm{GHz}$.

Methods. The spectrum of ${ }^{13} \mathrm{C}$ methylamine was recorded using conventional absorption spectrometers. The analysis of the rotational spectrum of ${ }^{13} \mathrm{C}$ methylamine in the ground vibrational state was performed on the basis of the group-theoretical high-barrier tunneling Hamiltonian that was developed for methylamine. The available multiple observations of the parent methylamine species toward Sgr $\mathrm{B} 2(\mathrm{~N})$ at 1, 2, and $3 \mathrm{~mm}$ using the Submillimeter Telescope and the $12 \mathrm{~m}$ antenna of the Arizona Radio Observatory were used to make a search for interstellar ${ }^{13} \mathrm{CH}_{3} \mathrm{NH}_{2}$.

Results. In the recorded spectra, we have assigned 2721 rotational transitions that belong to the ground vibrational state of the ${ }^{13} \mathrm{CH}_{3} \mathrm{NH}_{2}$. These measurements were fitted to the Hamiltonian model that uses 75 parameters to achieve an overall weighted rms deviation of 0.73 . On the basis of these spectroscopic results, predictions of transition frequencies in the frequency range up to $950 \mathrm{GHz}$ with $J \leq 50$ and $K_{\mathrm{a}} \leq 20$ are presented. The search for interstellar ${ }^{13} \mathrm{C}$ methylamine in available observational data was not successful and therefore only an upper limit of $6.5 \times 10^{14} \mathrm{~cm}^{-2}$ can be derived for the column density of ${ }^{13} \mathrm{CH}_{3} \mathrm{NH}_{2}$ toward $\mathrm{Sgr}$ B2(N), assuming the same source size, temperature, linewidth, and systemic velocity as for parent methylamine isotopic species.
\end{abstract}

Key words. ISM: molecules - methods: laboratory: molecular - submillimeter: ISM - molecular data - line: identification

\section{Introduction}

This paper is a part of a series of studies conducted in PhLAM Lille (France) that are devoted to the investigations of the spectra of different isotopic species of astrophysical molecules (Demyk et al. 2007; Margulès et al. 2009a,b, 2015; Carvajal et al. 2009; Tercero et al. 2012; Bouchez et al. 2012; Coudert et al. 2012; Richard et al. 2012, 2013; Haykal et al. 2013; Kutsenko et al. 2013; Nguyen et al. 2013). In particular these works led to the first interstellar detection of $\mathrm{HCOOCH}_{2} \mathrm{D}$ (Coudert et al. 2013), $\mathrm{HCOO}^{13} \mathrm{CH}_{3}$ (Carvajal et al. 2009), $\mathrm{HCO}^{18} \mathrm{OCH}_{3}, \mathrm{HC}^{18} \mathrm{OOCH}_{3}$ (Tercero et al. 2012), $\mathrm{CH}_{2} \mathrm{DOCH}_{3}$ (Richard et al. 2013). In the current paper we focus our attention on the ${ }^{13} \mathrm{C}$ isotopologue of the methylamine molecule. Interstellar methylamine was first detected toward Sgr B2 at $3.5 \mathrm{~cm}$ (Fourikis et al. 1974) and at $3 \mathrm{~mm}$ (Kaifu et al. 1974). Later surveys conducted by Turner (1989) toward Sgr B2(OH) and Nummelin et al. (1998) in Sgr B2(N) also found spectral lines of $\mathrm{CH}_{3} \mathrm{NH}_{2}$. Recently, methylamine has been detected in a spiral galaxy with a high redshift of 0.89, located in front of the quasar PKS 1830-211 (Muller et al. 2011). Also Fourikis et al. (1977) reported probable detection of deuterated methyl amine $\left(\mathrm{CH}_{3} \mathrm{NHD}\right)$ in the southern region of Sgr B2.

\footnotetext{
* Full Tables 3 and 4 are only available at the CDS via anonymous ftp to cdsarc.u-strasbg. fr $(130.79 .128 .5)$ or via http://cdsarc.u-strasbg.fr/viz-bin/qcat?]/A+A/587/A152
}

The rotational spectrum of the parent methylamine species has been the subject of a number of investigations. The latest paper on this issue (Motiyenko et al. 2014) covers the frequency range up to $2.6 \mathrm{THz}$ and the range of rotational quantum number $J$ up to 45 , which provides reliable predictions of the methylamine spectrum in the frequency range up to $3 \mathrm{THz}$. Also a number of deuterated isotopologues were studied by means of microwave spectroscopy: $\mathrm{CH}_{3} \mathrm{ND}_{2}$ (Takagi \& Kojima 1971), $\mathrm{CD}_{3} \mathrm{NH}_{2}$ (Sastry 1960; Kreglewski et al. 1990b), $\mathrm{CD}_{3} \mathrm{ND}_{2}$ (Lide 1954; Kreglewski et al. 1990a), $\mathrm{CH}_{3}$ NHD (Ohashi et al. 1991), and $\mathrm{CH}_{2} \mathrm{DNH}_{2}$ (Tamagake \& Tsuboi 1974). To our knowledge, there has been no previous spectroscopic study of ${ }^{13} \mathrm{CH}_{3} \mathrm{NH}_{2}$ isotopologue of methylamine in the microwave range. In this context, we present here an experimental study of the ${ }^{13} \mathrm{CH}_{3} \mathrm{NH}_{2}$ ground state rotational spectrum in the frequency range from 48 to $945 \mathrm{GHz}$. Based on the results obtained, a search for interstellar ${ }^{13} \mathrm{C}$ methylamine was made using available data from our recent observations of methanimine $\left(\mathrm{CH}_{2} \mathrm{NH}\right)$ and methyl amine $\left(\mathrm{CH}_{3} \mathrm{NH}_{2}\right)$ toward Sgr B2(N) (Halfen et al. 2013).

\section{Experiments}

The measurements in Lille were done with a ${ }^{13} \mathrm{C}$ enriched sample of methylamine purchased from Sigma-Aldrich, which was used without further purification. The measurements in the frequency ranges 150-315, 400-630 and 775-945 GHz were 
covered using the Lille spectrometer with solid state multiplied sources. The frequency of the Agilent synthesizer (12.5$17.5 \mathrm{GHz}$ ) was used as a reference source of radiation, which was further multiplied using various active and passive multipliers. Estimated uncertainties for measured line frequencies are $30 \mathrm{kHz}, 60 \mathrm{kHz}$, and $100 \mathrm{kHz}$, depending on the observed S/N ratio and the frequency range. The measurements in Kharkov were made using a sample with a natural abundance of ${ }^{13} \mathrm{C}$ isotopologue of methylamine. The measurements were performed in the frequency range from 48 to $148 \mathrm{GHz}$ using the automated spectrometer of Institute of Radio Astronomy of NASU (Alekseev et al. 2012). An initial search for the ${ }^{13} \mathrm{CH}_{3} \mathrm{NH}_{2}$ ground state rotational transitions was made using the spectrum records from our previous study (Ilyushin et al. 2005). Later a number of transitions were remeasured with an increased signal accumulation to provide a better $\mathrm{S} / \mathrm{N}$ ratio. The estimated uncertainties for the measured line frequencies are $30 \mathrm{kHz}$ and $100 \mathrm{kHz}$, depending on the observed $\mathrm{S} / \mathrm{N}$ ratio.

\section{Assignment and analysis of the spectrum}

\subsection{Theoretical model}

In our current study, we used the phenomenological Hamiltonian model, which is based on the group-theoretical high-barrier tunneling formalism that was developed for methylamine by Ohashi \& Hougen (1987). Since rather complete descriptions of this formalism and its implementation in the case of the methylamine molecule have been already presented several times in the literature (Ohashi \& Hougen 1987; Ohashi et al. 1987; Ilyushin et al. 2005; Ilyushin \& Lovas 2007; Motiyenko et al. 2014), we do not repeat a general description here. The formalism appeared to be the most successful for fitting the rotational spectrum of methylamine in the ground state, as well as in the first excited torsional state, and therefore it was chosen for the analysis of the ${ }^{13} \mathrm{CH}_{3} \mathrm{NH}_{2}$ ground state rotational spectrum.

The Hamiltonian operator is defined as

$$
\begin{aligned}
H & =h_{v}+h_{j} J^{2}+h_{k} J_{z}^{2}+\left(f_{+} J_{+}^{2}+f_{-} J_{-}^{2}\right)+q J_{z} \\
& +\left(r_{+} J_{+}+r_{-} J_{-}\right)+\left[s_{+}\left(J_{+} J_{z}+J_{z} J_{+}\right)\right. \\
& \left.+s_{-}\left(J_{-} J_{z}+J_{z} J_{-}\right)\right]+\left(f_{+}^{(2)} J_{+}^{4}+f_{-}^{(2)} J_{-}^{4}\right) \\
& + \text { higher order terms }
\end{aligned}
$$

where "higher order terms" represent ordinary centrifugal distortion terms as well as the $J$ and $K$ dependences of the various tunneling splitting parameters. The physical meaning of different parameters in Hamiltonian (1) was briefly discussed in Motiyenko et al. (2014). The computer fitting program used in the present analysis of the rotational spectrum of ${ }^{13} \mathrm{C}$ methylamine was developed by N. Ohashi (Ohashi et al. 1987) and previously modified by V. Ilyushin, who added new Hamiltonian terms (Ilyushin et al. 2005; Motiyenko et al. 2014) and provided the line strength calculations (Ilyushin \& Lovas 2007). As in the case of the main isotopologue (Ilyushin et al. 2005; Motiyenko et al. 2014) we have made a separate analysis of the quadrupole hyperfine structure of ${ }^{13} \mathrm{C}$ methylamine transitions, which is present because of the nonzero nuclear quadrupole moment of nitrogen ${ }^{14} \mathrm{~N}$. We used the following hyperfine energy expression, which permits the calculation of the hyperfine splittings for each rotational transition:

$$
\begin{aligned}
& E_{h f}(I, J, F)=\left[\frac{1}{2} \chi+\left\langle J_{x}^{2}+J_{y}^{2}-2 J_{z}^{2}\right\rangle-\frac{1}{2} \chi-\left\langle J_{x}^{2}-J_{y}^{2}\right\rangle\right. \\
& \left.+\chi_{x z}\left\langle J_{x} J_{z}+J_{z} J_{x}\right\rangle\right] \frac{2 f(I, J, F)}{J(J+1)},
\end{aligned}
$$

where $\chi_{+}=-\chi_{z z}, \chi_{-}=\chi_{y y}-\chi_{x x}\left(\right.$ Kreglewski et al. 1990a); $\left\langle J_{x}^{2}\right\rangle$, $\left\langle J_{y}^{2}\right\rangle,\left\langle J_{z}^{2}\right\rangle,\left\langle J_{x} J_{z}+J_{z} J_{x}\right\rangle$ are the expectation values of the operators in the axis system of the effective Hamiltonian (1) used, and $f(I, J, F)$ is the Casimir function (Gordy \& Cook 1984). As in the case of the main methylamine isotopologue, the analysis of observed hyperfine structure produced a set of "hyperfine free" rotational transition frequencies which were further analyzed using the effective Hamiltonian (1).

\subsection{Assignment and analysis}

We started our analysis of recorded spectra from a search of $K_{\mathrm{a}}=0 R$-type transitions which, because of torsion-wagging large amplitude motion in the molecule, form a characteristic quartet of lines. This search was based on the assumption that the substitution of ${ }^{13} \mathrm{C}$ will not affect significantly the tunneling splittings in the molecule (in comparison to the main methylamine isotopologue) and that the corresponding quartets of $K_{\mathrm{a}}=0 R$-type transitions will have a similar appearance in ${ }^{13} \mathrm{CH}_{3} \mathrm{NH}_{2}$ and ${ }^{12} \mathrm{CH}_{3} \mathrm{NH}_{2}$ spectra. The tentative assignment of the first such quartet at $173.2 \mathrm{GHz}$ that corresponds to the $K_{\mathrm{a}}=0$ $J=4 \leftarrow 3$ transitions gave us an opportunity to assign the whole series of this kind of transitions and get an initial approximation for the rotational constants of ${ }^{13} \mathrm{CH}_{3} \mathrm{NH}_{2}$ (at this stage the tunneling parameters were kept fixed at the values of the main methylamine isotopologue, Motiyenko et al. 2014). Obtained in this way, the initial model allowed us to continue assigning the spectra in a usual iterative manner by adding new assigned lines to the fit, refining the Hamiltonian model, and producing new predictions.

The Doppler-limited resolution of our spectrometers provided an opportunity to resolve nuclear quadrupole hyperfine splittings for 898 rotational transitions. Typically, as for the main methylamine isotopologue, a resolved pattern of the hyperfine structure was observed as a doublet with an approximately 2-to1 ratio in intensities. The stronger doublet component contains unresolved hyperfine transitions with selection rules $F=J+1 \rightarrow$ $F^{\prime}=J^{\prime}+1$ and $F=J-1 \rightarrow F^{\prime}=J^{\prime}-1$, whereas the weaker doublet component corresponds to the $F=J \rightarrow F^{\prime}=J^{\prime}$ transition. At the same time, a number of hyperfine components with selection rules $\Delta F \neq \Delta J$ were also assigned. To provide the hyperfinefree frequencies we used in our analysis of the rotation-torsionwagging spectrum of the ground state of ${ }^{13} \mathrm{C}$ methylamine, we fitted the frequencies of the individual hyperfine components using the model described above. The quadrupole hyperfine parameters $\chi_{+}$, and $\chi_{-}$that we obtained from the fitting of the hyperfine patterns of the rotational transitions are presented in Table 1.

In total, the dataset for the rotational spectra of ${ }^{13} \mathrm{CH}_{3} \mathrm{NH}_{2}$ in the ground vibrational state includes 2721 rotational transitions with an upper value of $J=43$. This set of rotational transitions was fitted to the effective Hamiltonian of the group-theoretical high-barrier tunneling formalism that was developed for methylamine by Ohashi \& Hougen (1987). The fit adopted in the present study as the "best" achieved the rootmean-square (rms) deviation of $0.039 \mathrm{MHz}$ that corresponds to the weighted rms deviation of 0.73 . The Hamiltonian model includes 75 parameters. The values of the molecular parameters obtained from the final fit are presented in Table 1. In Table 2, the main rotational and torsion-wagging splitting parameters for ${ }^{13} \mathrm{CH}_{3} \mathrm{NH}_{2}$ and ${ }^{12} \mathrm{CH}_{3} \mathrm{NH}_{2}$ (Motiyenko et al. 2014) are compared. From the comparison we see that the values of the main tunneling splitting parameters in both isotopologues of 
Table 1. Molecular parameters of the ground torsional state of ${ }^{13} \mathrm{CH}_{3} \mathrm{NH}_{2}$.

\begin{tabular}{|c|c|c|c|c|c|}
\hline \multicolumn{2}{|r|}{ Rotational } & \multicolumn{2}{|r|}{ Inversion } & \multicolumn{2}{|r|}{ Torsional } \\
\hline $\bar{B}$ & $21661.952564(88)$ & $h_{2}$ & $-1546.22791(71)$ & $h_{3}$ & $-2488.87759(96)$ \\
\hline$A-\bar{B}$ & $81496.38707(41)$ & $h_{4}$ & $2.72870(73)$ & $h_{5}$ & $2.87728(52)$ \\
\hline$B-C$ & $838.138853(57)$ & $h_{2 J}$ & $9.97182(41) \times 10^{-2}$ & $h_{7}$ & $-6.73(34) \times 10^{-3}$ \\
\hline$\Delta_{J}$ & $3.806084(18) \times 10^{-2}$ & $h_{2 K}$ & $1.737675(73)$ & $h_{3 J}$ & $-5.20711(85) \times 10^{-2}$ \\
\hline$\Delta_{J K}$ & $0.1668639(18)$ & $h_{4 J}$ & $-8.57(24) \times 10^{-5}$ & $h_{5 J}$ & $1.325(30) \times 10^{-4}$ \\
\hline$\Delta_{K}$ & $0.706766(12)$ & $h_{4 K}$ & $-4.400(40) \times 10^{-3}$ & $h_{3 K}$ & $1.165201(77)$ \\
\hline$\delta_{J}$ & $1.660274(31) \times 10^{-3}$ & $h_{2 J J}$ & $-3.674(11) \times 10^{-6}$ & $h_{5 K}$ & $-2.555(18) \times 10^{-3}$ \\
\hline$\delta_{K}$ & $-0.322295(13)$ & $h_{2 K K}$ & $-9.256(11) \times 10^{-4}$ & $h_{3 J J}$ & $-1.5021(18) \times 10^{-5}$ \\
\hline$\Phi_{J}$ & $-1.901(11) \times 10^{-8}$ & $h_{2 J K}$ & $-1.5092(20) \times 10^{-4}$ & $h_{3 K K}$ & $-2.283(10) \times 10^{-4}$ \\
\hline$\Phi_{J K}$ & $3.420(14) \times 10^{-6}$ & $h_{2 J K K}$ & $1.157(21) \times 10^{-7}$ & $h_{3 J K}$ & $-1.547(32) \times 10^{-5}$ \\
\hline$\Phi_{K J}$ & $-1.0297(59) \times 10^{-5}$ & $q_{2}$ & $21.53616(27)$ & $h_{5 J J}$ & $8.30(27) \times 10^{-8}$ \\
\hline$\Phi_{K}$ & $3.8535(94) \times 10^{-5}$ & $q_{4}$ & $-2.858(16) \times 10^{-2}$ & $h_{3 J J J}$ & $-2.72(12) \times 10^{-10}$ \\
\hline$\phi_{J}$ & $-9.25(34) \times 10^{-10}$ & $q_{2 J}$ & $-3.5835(14) \times 10^{-3}$ & $h_{3 J J K}$ & $-5.441(27) \times 10^{-8}$ \\
\hline$\phi_{J K}$ & $1.392(15) \times 10^{-6}$ & $q_{2 K}$ & $-1.9767(11) \times 10^{-2}$ & $f_{3}$ & $-0.165432(10)$ \\
\hline$\phi_{K}$ & $3.540(18) \times 10^{-4}$ & $q_{2 J J}$ & $4.955(84) \times 10^{-7}$ & $f_{5}$ & $3.632(27) \times 10^{-4}$ \\
\hline$L_{J K}$ & $4.589(48) \times 10^{-9}$ & $q_{2 J K}$ & $2.201(23) \times 10^{-6}$ & $f_{3 J}$ & $-3.191(22) \times 10^{-6}$ \\
\hline$L_{K K J}$ & $-1.730(18) \times 10^{-8}$ & $q_{2 K K}$ & $8.58(11) \times 10^{-6}$ & $f_{3 K}$ & $-2.153(32) \times 10^{-4}$ \\
\hline$\rho$ & $0.649733019(68)$ & $\begin{array}{l}f_{2} \\
f_{4}\end{array}$ & $\begin{array}{r}-9.22031(89) \times 10 \\
3.341(15) \times 10^{-4}\end{array}$ & $\begin{array}{l}f_{5 K} \\
f_{3}\end{array}$ & $\begin{array}{r}-6.52(19) \times 10^{-6} \\
-4.90(13) \times 10^{-10}\end{array}$ \\
\hline$\rho_{J}$ & $-1.952(59) \times 10^{-8}$ & $f_{2 J}$ & $5.087(14) \times 10^{-6}$ & $f_{3 J K}$ & $-2.161(81) \times 10^{-8}$ \\
\hline$\rho_{K}$ & $-1.1731(22) \times 10^{-6}$ & $f_{2 K}$ & $2.007(47) \times 10^{-4}$ & $f_{3}^{(2)}$ & $-3.5943(33) \times 10^{-6}$ \\
\hline$\rho_{J J}$ & $-4.04(16) \times 10^{-11}$ & $f_{4 K}$ & $-2.98(13) \times 10^{-6}$ & $f_{5}^{(2)}$ & $8.12(51) \times 10^{-8}$ \\
\hline \multirow[t]{3}{*}{$\rho_{J K}$} & $6.12(13) \times 10^{-10}$ & $f_{2 J J}$ & $-1.738(62) \times 10^{-10}$ & $s_{3}$ & $-0.3582(82)$ \\
\hline & & $f_{2}^{(2)}$ & $-3.9049(86) \times 10^{-6}$ & $r_{3}$ & $0.404(12)$ \\
\hline & Hyperfine & $f_{4}^{(2)}$ & $4.14(15) \times 10^{-8}$ & & \\
\hline$\chi_{+}$ & $-2.4154(33)$ & $f_{2 J}^{(2)}$ & $3.023(56) \times 10^{-10}$ & & \\
\hline \multirow[t]{3}{*}{$\chi_{-}$} & $6.0704(32)$ & $r_{2}$ & $10.584(26)$ & & \\
\hline & & $r_{2 J}$ & $-1.067(53) \times 10^{-3}$ & & \\
\hline & & $r_{2 K}$ & $-0.324(14)$ & & \\
\hline
\end{tabular}

Notes. All parameters are in MHz, except for $\rho, \rho_{J}, \rho_{K}, \rho_{J J}$, and $\rho_{J K}$ which are unitless. The structural parameter $\rho$ describes the coupling between the internal rotation of the methyl top and the overall rotation of the molecule as a whole. $\bar{B}=(B+C) / 2$.

Table 2. Main rotational and torsion-wagging splitting parameters (all in $\mathrm{MHz}$ except of $\rho$ which is unitless) of the ground torsional state of ${ }^{13} \mathrm{C}$ and ${ }^{12} \mathrm{C}$ methylamines.

\begin{tabular}{lcc}
\hline \hline Parameter $^{a}$ & ${ }^{13} \mathrm{CH}_{3} \mathrm{NH}_{2}$ & ${ }^{12} \mathrm{CH}_{3} \mathrm{NH}_{2}$ \\
\hline $\bar{B}$ & $21661.952564(88)$ & $22169.36697(12)$ \\
$A-\bar{B}$ & $81496.38707(41)$ & $80986.38521(57)$ \\
$B-C$ & $838.138853(57)$ & $877.88368(10)$ \\
$\Delta_{J}$ & $3.806084(18) \times 10^{-2}$ & $3.946663(19) \times 10^{-2}$ \\
$\Delta_{J K}$ & $0.1668639(18)$ & $0.1710869(21)$ \\
$\Delta_{K}$ & $0.706766(12)$ & $0.701310(14)$ \\
$\delta_{J}$ & $1.660274(31) \times 10^{-3}$ & $1.757561(60) \times 10^{-3}$ \\
$\delta_{K}$ & $-0.322295(13)$ & $-0.336039(16)$ \\
$h_{2}$ & $-1546.22791(71)$ & $-1549.18492(70)$ \\
$h_{3}$ & $-2488.87759(96)$ & $-2493.5110(12)$ \\
$h_{4}$ & $2.72870(73)$ & $2.72760(86)$ \\
$h_{5}$ & $2.87728(52)$ & $2.88853(58)$ \\
$q_{2}$ & $21.53616(27)$ & $21.54649(37)$ \\
$f_{2}$ & $-9.22031(89) \times 10^{-2}$ & $-9.6036(16) \times 10^{-2}$ \\
$f_{3}$ & $-0.165432(10)$ & $-0.173725(15)$ \\
$\rho$ & $0.649733019(68)$ & $0.649759856(98)$ \\
\hline
\end{tabular}

Notes. ${ }^{(a)} \bar{B}=(B+C) / 2$.

methylamine are quite close. Since the $A-\bar{B}$ is slightly larger in ${ }^{13} \mathrm{CH}_{3} \mathrm{NH}_{2}$ than in ${ }^{12} \mathrm{CH}_{3} \mathrm{NH}_{2}$, the strong $Q$-type series of lines in ${ }^{13} \mathrm{CH}_{3} \mathrm{NH}_{2}$ are shifted up in frequency compared with the main isotopologue as it is seen, for example, from Fig. 1, which presents the same $K_{\mathrm{a}}=6 \leftarrow 5{ }^{c} Q$ series of transitions as

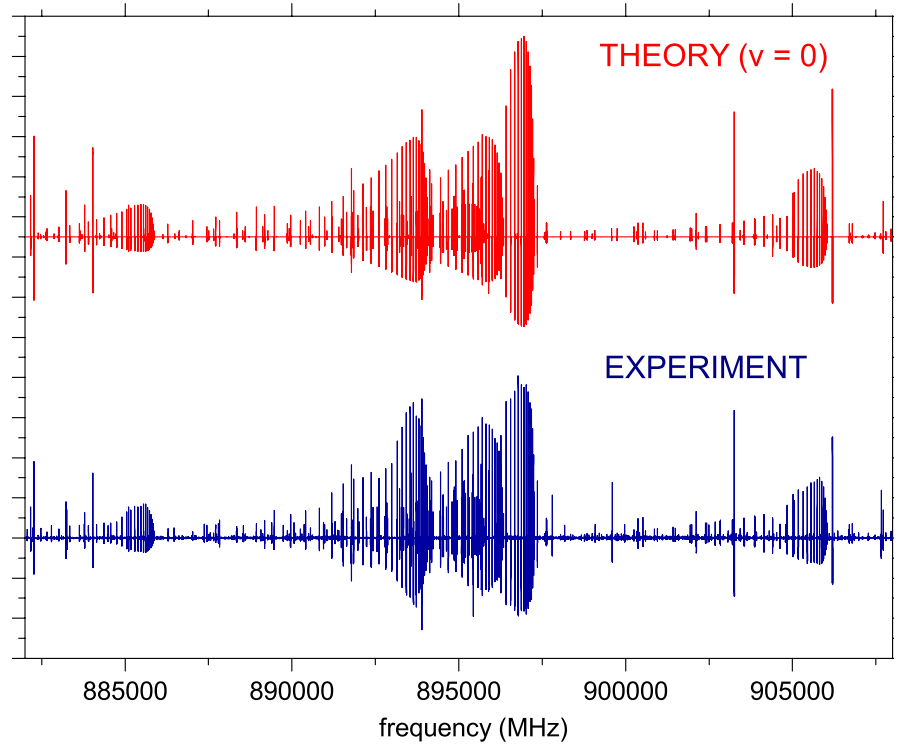

Fig. 1. Predicted (in red) and observed (in blue) rotational spectrum of ${ }^{13} \mathrm{C}$ methylamine between 882 and $908 \mathrm{GHz}$ dominated by ${ }^{c} Q$-type series of transitions with $K_{\mathrm{a}}=6 \leftarrow 5$. A slight inconsistency between predicted and observed spectrum, which may be visible for some strong lines, is due to source power and detector sensitivity variations. Weak unassigned lines are due to higher excited vibrational states.

in Fig. 1 of Motiyenko et al. (2014). A portion of the observed rotational spectrum of ${ }^{13} \mathrm{C}$ methylamine around $890 \mathrm{GHz}$, compared in Fig. 1 to the predicted rotational spectrum in the ground 
Table 3. Measured rotational transitions of ${ }^{13} \mathrm{CH}_{3} \mathrm{NH}_{2}$ in the ground vibrational state.

\begin{tabular}{|c|c|c|c|c|c|c|c|c|}
\hline$J^{\prime}$ & $K_{\mathrm{a}}^{\prime}$ & $\Gamma^{\prime}$ & $J^{\prime \prime}$ & $K_{\mathrm{a}}^{\prime \prime}$ & $\Gamma^{\prime \prime}$ & $\begin{array}{c}\text { Obs. freq. } \\
\text { (MHz) }\end{array}$ & $\begin{array}{c}\text { Uncertainty } \\
(\mathrm{MHz})\end{array}$ & $\begin{array}{c}\text { Obs.-calc. } \\
\text { (MHz) }\end{array}$ \\
\hline 13 & 1 & B1 & 13 & 0 & $\mathrm{~B} 2$ & 48242.931 & 0.030 & -0.0271 \\
\hline 12 & 1 & E2-1 & 12 & 0 & $\mathrm{E} 2+1$ & 48567.327 & 0.100 & 0.0139 \\
\hline 13 & 1 & E1-1 & 13 & 0 & $\mathrm{E} 1+1$ & 50719.336 & 0.030 & -0.0006 \\
\hline 31 & 5 & $\mathrm{E} 2+1$ & 30 & 6 & $\mathrm{E} 2+1$ & 448438.474 & 0.030 & 0.0174 \\
\hline 20 & 2 & $\mathrm{~A} 2$ & 19 & 3 & $\mathrm{~A} 1$ & 449166.832 & 0.060 & -0.0405 \\
\hline 24 & 2 & $\mathrm{E} 1+1$ & 24 & 1 & E1-1 & 449464.830 & 0.030 & 0.0117 \\
\hline 26 & 3 & $\mathrm{~A} 1$ & 26 & 2 & $\mathrm{~A} 2$ & 449514.293 & 0.030 & -0.0305 \\
\hline 13 & 7 & E1-1 & 14 & 6 & E1-1 & 449739.006 & 0.060 & 0.0465 \\
\hline 31 & 5 & $\mathrm{E} 1+1$ & 30 & 6 & $\mathrm{E} 1+1$ & 450025.342 & 0.030 & -0.0234 \\
\hline 13 & 7 & $\mathrm{E} 1+1$ & 14 & 6 & $\mathrm{E} 1+1$ & 450125.555 & 0.060 & -0.0034 \\
\hline \multicolumn{9}{|c|}{$\ldots$} \\
\hline
\end{tabular}

Notes. The full table is available at the CDS.

Table 4. Predicted transitions of ${ }^{13} \mathrm{C}$ methylamine in the ground vibrational state.

\begin{tabular}{ccccccccccccc}
\hline \hline$J^{\prime}$ & $K_{\mathrm{a}}^{\prime}$ & $\Gamma^{\prime}$ & $F^{\prime}$ & $J^{\prime \prime}$ & $K_{\mathrm{a}}^{\prime \prime}$ & $\Gamma^{\prime \prime}$ & $F^{\prime \prime}$ & $\begin{array}{c}\text { Calc. freq. } \\
(\mathrm{MHz})\end{array}$ & $\begin{array}{c}\text { Uncertainty } \\
(\mathrm{MHz})\end{array}$ & $\begin{array}{c}\mu^{2} S \\
\left(\mathrm{D}^{2}\right)\end{array}$ & $W_{s t}$ & $\begin{array}{c}E_{l} \\
\mathrm{~cm}^{-1}\end{array}$ \\
\hline 18 & 1 & B1 & 17 & 17 & 0 & B2 & 17 & 945375.3582 & 0.0126 & 0.011 & 3 & 220.416 \\
18 & 1 & $\mathrm{~B} 1$ & 18 & 17 & 0 & $\mathrm{~B} 2$ & 17 & 945375.4011 & 0.0124 & 3.432 & 3 & 220.416 \\
18 & 1 & $\mathrm{~B} 1$ & 19 & 17 & 0 & $\mathrm{~B} 2$ & 18 & 945376.9962 & 0.0124 & 3.629 & 3 & 220.416 \\
18 & 1 & $\mathrm{~B} 1$ & 18 & 17 & 0 & $\mathrm{~B} 2$ & 18 & 945377.0368 & 0.0126 & 0.011 & 3 & 220.416 \\
18 & 1 & $\mathrm{~B} 1$ & 17 & 17 & 0 & $\mathrm{~B} 2$ & 16 & 945377.0903 & 0.0124 & 3.246 & 3 & 220.416 \\
34 & 3 & $\mathrm{E} 1-1$ & 34 & 33 & 4 & $\mathrm{E} 1-1$ & 33 & 945404.9932 & 0.0237 & 3.703 & 3 & 853.710 \\
34 & 3 & $\mathrm{E} 1-1$ & 35 & 33 & 4 & $\mathrm{E} 1-1$ & 34 & 945405.4807 & 0.0237 & 3.814 & 3 & 853.710 \\
34 & 3 & $\mathrm{E} 1-1$ & 33 & 33 & 4 & $\mathrm{E} 1-1$ & 32 & 945405.4956 & 0.0237 & 3.596 & 3 & 853.710 \\
33 & 2 & $\mathrm{E} 2+1$ & 32 & 32 & 3 & $\mathrm{E} 2+1$ & 31 & 945502.5113 & 0.0310 & 3.362 & 1 & 786.807 \\
33 & 2 & $\mathrm{E} 2+1$ & 34 & 32 & 3 & E2+1 & 33 & 945502.5288 & 0.0310 & 3.572 & 1 & 786.807 \\
33 & 2 & $\mathrm{E} 2+1$ & 33 & 32 & 3 & E2+1 & 32 & 945503.1308 & 0.0310 & 3.465 & 1 & 786.807 \\
\hline
\end{tabular}

Notes. The full table is available at the CDS.

vibrational state, illustrates an overall very good correspondence between experimental and theoretical spectra. The majority of strong lines are assigned (and correspond mainly to ${ }^{c} Q$-type transitions with $K_{\mathrm{a}}=6 \leftarrow 5$ ) and are well predicted by our current model, although a number of rather strong unassigned lines, presumably belonging to the higher excited states, may still be found in the experimental spectrum.

The list of measured rotational transitions of the ground vibrational state of ${ }^{13} \mathrm{C}$ methylamine is presented in Table 3 . In the first six columns of Table 3, the quanta for each spectral line are given: $J, K_{\mathrm{a}}$, and symmetry label $\Gamma$. In the following columns we provide the observed transition frequencies, measurement uncertainties, and residuals from the fit. Only hyperfine free rotational frequencies that were used in the final fit are presented in Table 3 for the observed transitions. Table 4 gives the prediction of the ground state rotational spectrum of ${ }^{13} \mathrm{C}$ methylamine up to $950 \mathrm{GHz}$. The spectrum was calculated taking nuclear quadrupole hyperfine coupling into account. Therefore each energy level in Table 4 is labeled by four quantum numbers: $J, K_{\mathrm{a}}, \Gamma$, and total angular momentum $F$. The quantum numbers are followed by the columns with calculated transition frequencies and corresponding uncertainties. The next two columns contain the product $\mu^{2} S$ (where $\mu$ is the dipole moment of the molecule and $S$ is transition linestrength), and the nuclear spin statistical weight which is equal to 1 for $A_{1}, A_{2}$, and $E_{2}$ species and equal to 3 for $B_{1}, B_{2}$, and $E_{1}$ species. The next column represents the energy of the lower state. In our calculations of the ${ }^{13} \mathrm{CH}_{3} \mathrm{NH}_{2}$ spectrum, the values for the dipole moment components were taken to be equal to the corresponding values of the parent methylamine isotopologue $\mu_{\mathrm{a}}=-0.307 \mathrm{D}$ and $\mu_{c}=1.258 \mathrm{D}$ (Motiyenko et al. 2014). Owing to their significant sizes, the complete versions of Tables 3 and 4 are presented at the CDS. Here only parts of Tables 3 and 4 are provided for illustration purposes.

In our predictions of the ground state rotational spectrum, we have adopted the limitation of $J_{\max }=50, K_{\mathrm{a}, \max }=20$. In the calculation, we have included the rotational transitions with rotational selection rules $\Delta J=0, \pm 1$ and $\Delta K_{\mathrm{a}}=0, \pm 1, \pm 2, \pm 3$. In Table 4, those transitions that match the frequency range requirement (from $1 \mathrm{GHz}$ to $950 \mathrm{GHz}$ ), whose predicted uncertainties are less than $1 \mathrm{MHz}$ and whose line strength exceeds the limit of 0.01, are included. Also, to limit the size of Table 4, we have only presented the most intense hyperfine quadrupole components for which the relative intensities exceeded $0.1 \%$ of the total intensity of the rotation transition (i.e., mainly with the selection rule $\Delta F=\Delta J)$. In addition, we provide the rotational part of the partition function $Q_{\mathrm{r}}(T)$ of ${ }^{13} \mathrm{C}$ methylamine calculated from first principles (Table 5), i.e. via direct summation over the rotational-tunneling states. The maximum value of the $J$ quantum number for the energy levels taken for calculating the partition function is 100 . The vibrational part $Q_{\mathrm{v}}(T)$ may be estimated in the harmonic approximation using the normal modes reported by Schimanouchi (1972) for the main methylamine isotopologue. Simple formulas for calculating $Q_{\mathrm{V}}(T)$ can be found elsewhere (see, for example Gordy \& Cook 1984).

\section{Astronomical observations}

The spectral records used to search for ${ }^{13} \mathrm{CH}_{3} \mathrm{NH}_{2}$ here are a part of a complete spectral-line survey of the 1,2, and $3 \mathrm{~mm}$ 
Table 5. Rotation part $Q_{\mathrm{r}}(T)$ of the total internal partition function $Q(T)=Q_{\mathrm{v}}(T) \times Q_{\mathrm{r}}(T)$, calculated from first principles using the parameter set of Table 1 .

\begin{tabular}{cc}
\hline \hline Temperature $(\mathrm{K})$ & $Q_{\mathrm{r}}(T)$ \\
\hline 300 & 31908.65 \\
200 & 17356.87 \\
150 & 11269.17 \\
50 & 2165.81 \\
10 & 193.24 \\
\hline
\end{tabular}

windows toward Sgr B2(N). The data were recorded during the period September 2002 to March 2013 using the Arizona Radio Observatory (ARO) $12 \mathrm{~m}$ telescope on Kitt Peak and the Submillimeter Telescope (SMT) on Mount Graham.

The receivers employed were dual-polarization, SIS mixers covering the 3 and 2 mm bands (68-116 and 130-172 GHz). The single-sideband mixers were tuned to reject the image sideband of a level typically $\geq 18 \mathrm{~dB}$. Data were also obtained with a new dual-polarization receiver using ALMA Band $3(83-116 \mathrm{GHz})$ sideband-separating (SBS) mixers. With these devices, the image rejection was usually $\geq 16 \mathrm{~dB}$, attained within the mixer architecture. The temperature scale was determined by the chopper wheel method, corrected for forward spillover losses, and is given as $T_{\mathrm{R}}^{*}$. The radiation temperature $T_{\mathrm{R}}$, assuming the source only fills the main beam, is $T_{\mathrm{R}}=\frac{T_{\mathrm{R}}^{*}}{\eta_{\mathrm{c}}}$, where $\eta_{\mathrm{c}}$ is the main beam efficiency, corrected for forward spillover losses. The spectrometer backend utilized for the measurements was a millimeter autocorrelator (MAC) with either $390 \mathrm{kHz}$ or $781 \mathrm{kHz}$ resolution, and a bandwidth of $600 \mathrm{MHz}$ channel ${ }^{-1}$. The spectra were smoothed using a cubic spline routine to a $1 \mathrm{MHz}$ resolution.

At the SMT, observations in the frequency range 210$280 \mathrm{GHz}(1 \mathrm{~mm})$ were taken with a dual-polarization receiver, utilizing ALMA Band 6 SBS mixers with rejection of at least 16 $\mathrm{dB}$ of the image sideband. The temperature scale was determined by the chopper wheel method, and is given as $T_{\mathrm{A}}^{*}$. The radiation temperature $T_{\mathrm{R}}$ is $T_{\mathrm{R}}=\frac{T_{\mathrm{A}}^{*}}{\eta_{\mathrm{b}}}$, where $\eta_{\mathrm{b}}$ is the main beam efficiency. A 2048 channel filter bank with $1 \mathrm{MHz}$ resolution was utilized as the spectrometer backend, split into parallel mode $(2 \times 1024)$. The beam size ranged from $87^{\prime \prime}$ to $37^{\prime \prime}$ at the $12 \mathrm{~m}$ and $36^{\prime \prime}$ to $23^{\prime \prime}$ at the SMT. All observations were conducted toward Sgr B2(N) $\left(\alpha=17^{\mathrm{h}} 44^{\mathrm{m}} 09.5^{\mathrm{s}} ; \delta=-28^{\circ} 21^{\prime} 20^{\prime \prime} ; \mathrm{B} 1950.0\right.$, or $\alpha=17^{\mathrm{h}} 47^{\mathrm{m}} 19.2^{\mathrm{s}} ; \delta=-28^{\circ} 22^{\prime} 22^{\prime \prime} ; \mathrm{J} 2000.0$ : $\left.\mathrm{NED}^{1}\right)$ in position switching mode with a $+30^{\prime}$ OFF position in azimuth. A 10-20 MHz local oscillator shift and direct observation of the image sideband were employed to identify any image contamination. The pointing accuracy is estimated to be $\pm 5^{\prime \prime}-10^{\prime \prime}$ at the $12 \mathrm{~m}$ and $\pm 2^{\prime \prime}$ at the SMT. The telescope pointing was determined by observations of planets.

\section{Column density upper limit toward Sgr B2(N)}

We searched for the transitions of ${ }^{13} \mathrm{CH}_{3} \mathrm{NH}_{2}$ in our complete spectral-line survey of the 1,2 , and $3 \mathrm{~mm}$ windows toward $\mathrm{Sgr}$ $\mathrm{B} 2(\mathrm{~N})$. The search was conducted in a systematic way by modeling the expected emission in the local thermodynamic equilibrium (LTE) approximation, which is certainly valid for the rotational transitions in the vibrational ground state. There are a number of non-LTE effects which may affect our results. We may overestimate a total column density of ${ }^{13} \mathrm{CH}_{3} \mathrm{NH}_{2}$ gas if the

\footnotetext{
1 http://ned.ipac.caltech.edu/forms/calculator.html
}

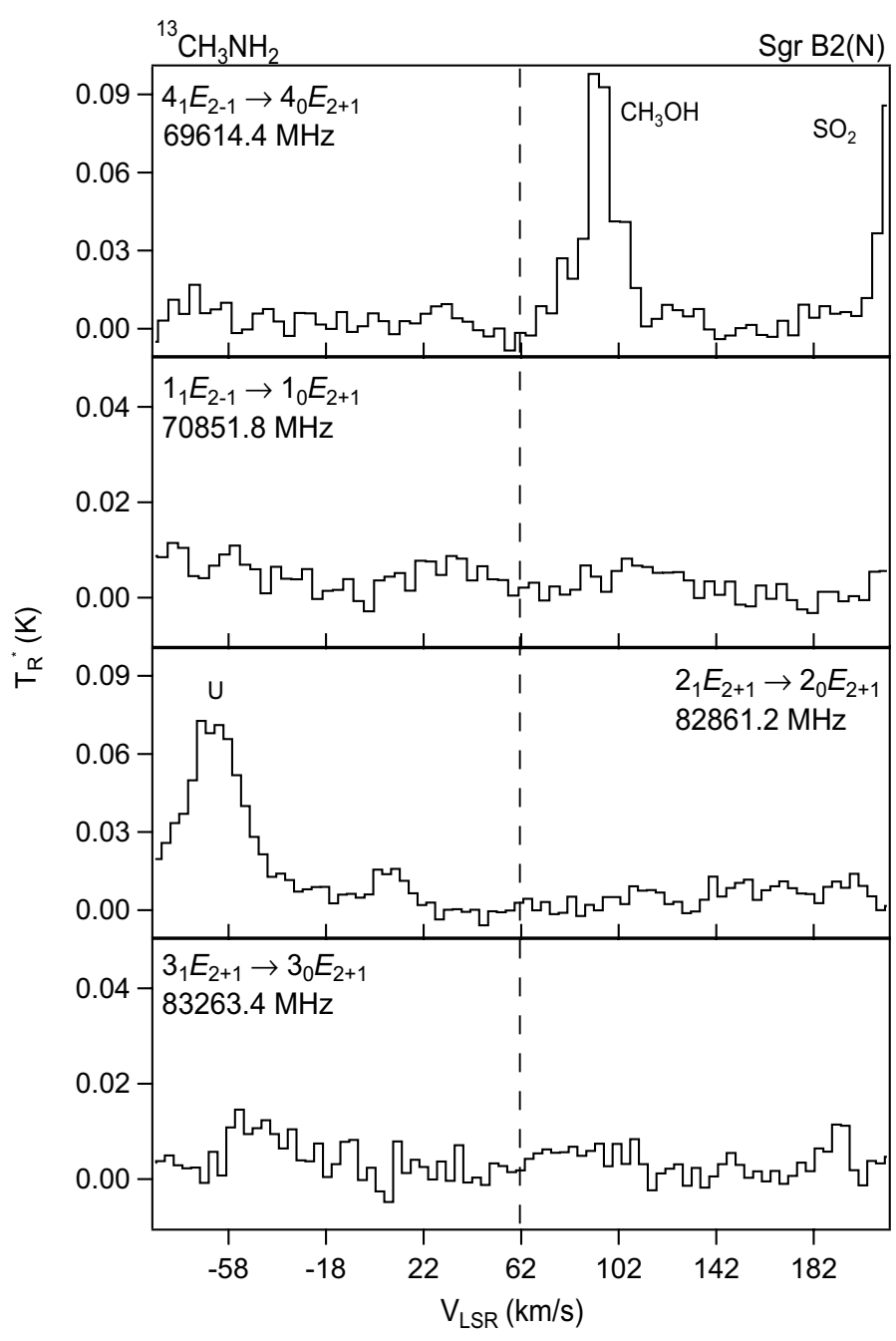

Fig. 2. Results of an interstellar search for the selected rotational transitions of ${ }^{13} \mathrm{CH}_{3} \mathrm{NH}_{2}$.

density is not sufficient to thermally populate the vibrationally excited states via collisions (the partition function of ${ }^{13} \mathrm{C}$ methylamine includes the contribution of excited vibrational states in the LTE approximation). At the same time, we may underestimate the total column density because infrared excitation may also contribute to the population of the vibrationally excited states (e.g., Nummelin \& Bergman 1999). Also we cannot exclude that radiative decay, involving rovibrational transitions, may affect the rotational populations of the vibrational ground state. Since there is no easy way to correct for these possible non-LTE effects, we restrained our analysis to the LTE assumption. Within the frequency ranges of our survey, we have looked for 69 transitions of ${ }^{13} \mathrm{C}$ methylamine that are predicted to be the most prominent spectral features of the molecule under the physical parameters of our model. We assumed the same source size, temperature, linewidth, and systemic velocity as for parent isotopic species of methylamine (Halfen et al. 2013). The physical parameters of the ${ }^{13} \mathrm{CH}_{3} \mathrm{NH}_{2}$ model are $T_{\text {rot }}=159 \mathrm{~K}$, $\Delta V_{1 / 2}=15 \mathrm{~km} \mathrm{~s}^{-1}$, and $V_{\mathrm{LSR}}=64 \mathrm{~km} \mathrm{~s}^{-1}$ with the assumption that the source fills the beam. Among checked transitions, only eight are found to be relatively free of contamination, and these are listed in Table 6. All other transitions of ${ }^{13} \mathrm{CH}_{3} \mathrm{NH}_{2}$ are heavily blended with transitions of other species and cannot be detected with our single-dish data. The LTE modeled spectrum for the four selected contamination-free transitions of ${ }^{13} \mathrm{CH}_{3} \mathrm{NH}_{2}$ is compared to the observed spectrum in Fig. 2. It 
Table 6. Uncontaminated transitions of ${ }^{13} \mathrm{C}$ methylamine in Sgr B2(N).

\begin{tabular}{ccccccccccc}
\hline \hline$J^{\prime}$ & $K_{\mathrm{a}}^{\prime}$ & $\Gamma^{\prime}$ & $J^{\prime \prime}$ & $K_{\mathrm{a}}^{\prime \prime}$ & $\Gamma^{\prime \prime}$ & $\begin{array}{c}\text { Frequency } \\
(\mathrm{MHz})\end{array}$ & $\eta_{\mathrm{c}}$ & $\begin{array}{c}T_{\mathrm{R}}^{*} \\
(\mathrm{~K})\end{array}$ & $\begin{array}{c}E_{u} \\
(\mathrm{~K})\end{array}$ & $\begin{array}{c}\mu^{2} S \\
\left(\mathrm{D}^{2}\right)\end{array}$ \\
\hline 4 & 1 & $\mathrm{E} 2-1$ & 4 & 0 & $\mathrm{E} 2+1$ & 69614.44 & 0.94 & $<0.005$ & 24.72 & 5.75 \\
2 & 1 & $\mathrm{E} 2-1$ & 2 & 0 & $\mathrm{E} 2+1$ & 70741.04 & 0.94 & $<0.004$ & 10.22 & 2.40 \\
1 & 1 & $\mathrm{E} 2-1$ & 1 & 0 & $\mathrm{E} 2+1$ & 70851.75 & 0.94 & $<0.003$ & 6.06 & 1.27 \\
6 & 1 & $\mathrm{E} 1-1$ & 6 & 0 & $\mathrm{E} 1+1$ & 73461.33 & 0.93 & $<0.005$ & 47.62 & 10.61 \\
2 & 1 & $\mathrm{~B} 2$ & 2 & 0 & $\mathrm{~B} 1$ & 78707.52 & 0.92 & $<0.004$ & 10.32 & 3.98 \\
3 & 1 & $\mathrm{E} 1-1$ & 3 & 0 & $\mathrm{E} 1+1$ & 78740.49 & 0.92 & $<0.004$ & 16.70 & 4.79 \\
2 & 1 & $\mathrm{E} 2+1$ & 2 & 0 & $\mathrm{E} 2+1$ & 82861.25 & 0.92 & $<0.003$ & 10.80 & 1.58 \\
3 & 1 & $\mathrm{E} 2+1$ & 3 & 0 & $\mathrm{E} 2+1$ & 83263.42 & 0.92 & $<0.003$ & 17.06 & 1.71 \\
\hline
\end{tabular}

is seen that, at the typical noise level of this survey, we have the apparent non-detection of these ${ }^{13} \mathrm{CH}_{3} \mathrm{NH}_{2}$ transitions toward Sgr B2(N). Therefore only an upper limit on the column density of ${ }^{13} \mathrm{CH}_{3} \mathrm{NH}_{2}$ can be deduced from our current observational data which, for the assumed physical parameters of our model, is $<6.5 \times 10^{14} \mathrm{~cm}^{-2}$.

\section{Conclusions}

The first study of the rotational spectra of the ${ }^{13} \mathrm{C}$ isotopologue of methylamine molecule was carried out in the frequency range from 48 to $945 \mathrm{GHz}$. Using the effective Hamiltonian model based on the group-theoretical high-barrier tunneling formalism developed for methylamine by Ohashi \& Hougen (1987), we were able to fit the available data within experimental accuracy. The results of the present study allow us to produce reliable predictions of rotational spectra in the ground vibrational state of ${ }^{13} \mathrm{CH}_{3} \mathrm{NH}_{2}$ for astrophysical purposes in the frequency range up to $950 \mathrm{GHz}$ for $0<J<50$ and $0<K_{\mathrm{a}}<20$. An attempt to detect interstellar ${ }^{13} \mathrm{CH}_{3} \mathrm{NH}_{2}$ toward Sgr B2(N) using available observational data from a spectral-line survey of the 1,2 , and $3 \mathrm{~mm}$ windows using the Arizona Radio Observatory (ARO) $12 \mathrm{~m}$ telescope on Kitt Peak and the Submillimeter Telescope (SMT) on Mount Graham was not successful and resulted in the determination of the ${ }^{13} \mathrm{CH}_{3} \mathrm{NH}_{2}$ column density upper limit of $<6.5 \times 10^{14} \mathrm{~cm}^{-2}$. The ratio of ${ }^{12} \mathrm{CH}_{3} \mathrm{NH}_{2}$ to ${ }^{13} \mathrm{CH}_{3} \mathrm{NH}_{2}$, using the column density of ${ }^{12} \mathrm{CH}_{3} \mathrm{NH}_{2}$ from Halfen et al. (2013), is >7.7. This value can be compared to those from other species, such as $\mathrm{HC}_{3} \mathrm{~N}$ and $\mathrm{CH}_{2} \mathrm{CHCN}$, in Sgr B2(N) of 20 (Belloche et al. 2013, Halfen et al., in prep.). Thus, the upper limit for ${ }^{13} \mathrm{CH}_{3} \mathrm{NH}_{2}$ in Sgr B2(N) could be approximately $2.5 \times 10^{14} \mathrm{~cm}^{-2}$, about a factor of three less than the current limit, and below the current detection limit. We hope that the new observations toward Sgr B2(N) using the very sensitive ALMA telescope will provide an opportunity to detect ${ }^{13} \mathrm{CH}_{3} \mathrm{NH}_{2}$ in the interstellar medium.

Acknowledgements. The authors are indebted to Dr. N. Ohashi for providing his fitting program for methylamine. This work was done with the support of the Ukrainian-French CNRS-PICS 6051 project. This research was supported by NSF grants AST-1140030 and AST-1211502. The SMT and Kitt Peak $12 \mathrm{~m}$ are operated by the Arizona Radio Observatory (ARO), Steward Observatory, University of Arizona, with support from the NSF University Radio Observatories program (URO: AST-1140030).

\section{References}

Alekseev, E. A., Motiyenko, R. A., \& Margulès, L. 2012, Radio Physics and Radio Astronomy, 3, 75

Belloche, A., Müller, H. S. P., Menten, K. M., Schilke, P., \& Comito, C. 2013, A\&A, 559, A47

Bouchez, A., Margulès, L., Motiyenko, R. A., et al. 2012, A\&A, 540, A51

Carvajal, M., Margulès, L., Tercero, B., et al. 2009, A\&A, 500, 1009

Demaison, J., Wlodarczak, G., Huet, T. R., et al. 2009, A\&A, 500, 1009

Coudert, L. H., Margulès, L., Huet, T. R., et al. 2012, A\&A, 543, A46

Coudert, L. H., Drouin, B. J., Tercero, B., et al. 2013, ApJ, 779, A119

Demyk, K., Mäder, H., Tercero, B., et al. 2007, A\&A, 466, 255

Fourikis, N., Takagi, K., \& Morimoto, M. 1974, ApJ, 191, L139

Fourikis, N., Takagi, K., \& Saito, S. 1977, ApJ, 212, L33

Gordy, W., \& Cook, R. L. Microwave 1984, Molecular Spectra, Techniques of Chemistry, Vol. XVIII (New York: Wiley)

Halfen, D. T., Ilyushin, V. V., \& Ziurys, L. M. 2013, ApJ, 767, 66

Haykal, I., Motyienko, R. A., Margulès, L., \& Huet, T. R. 2013, A\&A, 549, A96

Ilyushin, V., \& Lovas, F. J. 2007, J. Phys. Chem. Ref. Data, 36, 1141

Ilyushin, V. V., Alekseev, E. A., Dyubko, S. F., Motiyenko, R. A., \& Hougen, J. T. 2005, J. Mol. Spec., 229, 170

Kaifu, N., Morimoto, M., Nagane, K., et al. 1974, ApJ, 191, L135

Kreglewski, M., Stryjewski, D., \& Dreizler, H. 1990a, J. Mol. Spec., 139, 182

Kreglewski, M., Jäger, W., \& Dreizler, H. 1990b, J. Mol. Spec., 144, 334

Kutsenko, A. S., Motiyenko, R. A., Margulès, L., \& Guillemin, J.-C. 2013, A\&A, 549, A 128

Lide, Jr., D. R. 1954, J. Chem. Phys., 22, 1613

Margulès, Motiyenko, R., Demyk, K., Tercero, B. 2009a, A\&A, 493, 565

Margulès, L., Coudert, L. H., Møllendal, H., et al. 2009b, J. Mol. Spec., 254, 55

Margulès, L., Motiyenko, R. A., Ilyushin, V. V., \& Guillemin, J.-C. 2015, A\&A, 579, A46

Motiyenko, R. A., Ilyushin, V. V., Drouin, B. J., Yu, S., \& Margulès, L. 2014, A\&A, 563, A137

Muller, S., Beelen, A., Guelin, M., et al. 2011, A\&A, 535, A103

Nguyen, L., Walters, A., Margulès, L., et al. 2013, A\&A, 553, A84

Nummelin, A., \& Bergman, P. 1999, A\&A, 341, L59

Nummelin, A., Bergman, P., Hjalmarson, A., et al. 1998, ApJS, 117, 427

Ohashi, N., \& Hougen, J. T. 1987, J. Mol. Spec., 121, 474

Ohashi, N., Takagi, K., Hougen, J. T., Olson, W. B., \& Lafferty, W. J. 1987, J. Mol. Spec., 126, 443

Ohashi, N., Oda, M., \& Takagi, K. 1991, J. Mol. Spec., 145, 180

Richard, C., Margulès, L., Motiyenko, R. A., \& Guillemin, J.-C. 2012, A\&A, 543, A 135

Richard, C., Margulès, L., Caux, E., et al. 2013, A\&A, 552, A117

Sastry, K. V. L. N. 1960, Proc. Indian Acad. Sci. Sect. A, 51, 301

Schimanouchi, T. 1972, in Tables of Molecular Vibrational Frequencies, Vol. I: consolidated (Washington, DC: National Bureau of Standards), 67

Takagi, K., \& Kojima, T. 1971, J. Phys. Soc. Jpn., 30, 1145

Tamagake, K., \& Tsuboi, M. 1974, J. Mol. Spec., 53, 204

Tercero, B., Margulès, L., Carvajal, M., et al. 2012, A\&A, 538, A119

Turner, B. E. 1989, ApJS, 70, 539 